\title{
External Multicenter Validation of the Mehran Risk Score for Contrast Induced Acute Kidney Injury
}

\author{
Nashwa Abdulsalam* \\ Department of Cardiac Electrophysiology, University of Nebraska Medical Center, USA
}

*Corresponding author: Nashwa Abdulsalam, Department of Cardiac electrophysiology, University of Nebraska Medical Center, 42nd and Emile, Omaha, NE 68198, USA

\begin{abstract}
Background: Contrast induced acute kidney injury (CIAKI) is a known complication of percutaneous coronary intervention ( $\mathrm{PCl}$ ). Mehran Risk Score (MR score) has been previously shown to predict CIAKI, renal replacement therapy (RRT), and one-year mortality in patients undergoing $\mathrm{PCl}$. The purpose of our study was to externally validate the MR score.

Methods: To examine the utility of the MR score we reviewed records of 931 adult patients who underwent $\mathrm{PCl}$ in 2005 at 3 academic medical centers. Patients with acute myocardial infarction, end stage renal disease and contrast exposure within one week of $\mathrm{PCl}$ were excluded. MR score was calculated for each patient and stratified into 4 groups: MR score 0-5 (group 1), 6-10 (group 2), 11-15 (group 3), $\geq$ 16 (group 4). CIAKI was defined as an increase in serum creatinine of $25 \%$ or $0.5 \mathrm{mg} / \mathrm{dl}$ over baseline 48 hours post $\mathrm{PCl}$. Need for hemodialysis was assessed within 1 month after $\mathrm{PCl}$. All-cause mortality was assessed 1 year after $\mathrm{PCl}$. Likelihood ratio was calculated to assess the MR score discrimination for our data as well as Mehran, et al.
\end{abstract}

Results: The overall incidence of CIAKI, hemodialysis and mortality were $12.2 \%, 0.4 \%$, and $9.0 \%$ respectively. A higher MR score was strongly associated with development of CIAKI and mortality ( $p<0.01$ for trend). There was no difference in the rate of CIAKI overall or in each MR score group when the 2 populations were compared, however, the risk of death was higher in our population (RR 1.58, Cl 1.37$1.89, p<0.001$ )

Conclusion: In conclusion, we were able to externally validate the MR score as a useful tool to predict CIAKI and one-year all-cause mortality post $\mathrm{PCl}$.

\section{Keywords}

Contrast induced acute kidney injury, Percutaneous coronary intervention, Mehran risk score

\section{Introduction}

Contrast-induced acute kidney injury (CIAKI) occurs in approximately $7 \%$ of patients undergoing percutaneous coronary intervention (PCI) [1]. Its incidence varies depending on the definition used and the cohort studied. $\mathrm{CIAKI}$ is associated with increased length $[2,3]$ and cost of in-hospital stay [4], and increased mortality [5,6]. Despite identifying several risk factors and instituting preventive measures, $\mathrm{PCl}$ still remains a common cause of hospital-acquired acute kidney injury (AKI) [7]. Several clinical trials have aimed to reduce CIAKI by use of agents such as normal saline, $0.45 \%$ saline, $\mathrm{N}$-acetylcysteine, theophylline, fenoldopam, dopamine, and furosemide [8]. However, to date no agent has proven to be effective in the prevention of CIAKI $[8,9]$. $A$ risk predicting score that helps in identifying patients at increased risk of developing CIAKI may aid in targeted application of these therapies to better test their effectiveness.

Mehran, et al. derived a risk score (MR score) for predicting CIAKI post $\mathrm{PCl}$ from a prospective interventional cardiology database. MR score is simple, accounts for the cumulative nature of risk assessments based on weighted integers and had a robust development dataset $(N=5,571)$ that predicted risk for developing $\mathrm{ClAKI}$, need for renal replacement therapy (RRT) and one-year mortality [10] (Table 1). Risk predicting scores developed in other contexts such as acute kidney injury following cardiac surgery have failed to show similar accuracy in external cohorts than when they were initially developed [11]. Thus, externally validating the Mehran risk score (MRS) 
Table 1: The mehran risk score.

\begin{tabular}{|l|l|}
\hline Risk Factor & Points \\
\hline Hypotension* & 5 \\
\hline Intra-aortic balloon pump & 5 \\
\hline Congestive heart failure† & 5 \\
\hline Age $>75$ years & 4 \\
\hline Anemia ${ }^{\ddagger}$ & 3 \\
\hline Diabetes & 3 \\
\hline Contrast volume & 1 for each $100 \mathrm{cc}$ \\
\hline $\begin{array}{l}\text { Chronic kidney disease } \\
\text { Serum creatinine }>1.5 \mathrm{mg} / \\
\text { dL or } \\
\text { GFR }<60 \mathrm{ml} / \mathrm{min} / 1.73 \mathrm{~m}^{2}\end{array}$ & 4 \\
& 2 for GFR $40-59$ \\
4 for GFR $20-39$ \\
6 for GFR $<20$
\end{tabular}

"Systolic blood pressure $<80 \mathrm{mmHg}$ for at least one hour requiring inotropic support with medications or IABP within 24 hours periprocedurally; ${ }^{\dagger} \mathrm{NYHA}$ functional class III or IV and/or history of pulmonary edema; ${ }^{\ddagger}$ Hematocrit $<39 \%$ for men or $<$ $36 \%$ for women.

serves as an important tool for clinicians and patients to make informed decisions and further generalize it applicability. We undertook a multi-center validation of this score among patients undergoing non-emergent $\mathrm{PCl}$ from across three different academic institutions.

\section{Methods}

We reviewed all records of patients ages $>18$ years who underwent $\mathrm{PCl}$ in 2005 at three hospitals, Strong Memorial Hospital/University of Rochester, Rochester, NY; Rochester General Hospital, Rochester, NY; and Memorial Medical Center/Southern Illinois University, Springfield, Illinois. The study was approved by the respective institutional review boards. Patients with baseline serum creatinine and a serum creatinine at 48 hours post- $\mathrm{PCl}$ were included. Patients were excluded if they presented with an acute myocardial infarction, had end stage renal disease on dialysis, were exposed to intravenous contrast within one week of $\mathrm{PCl}$, or did not have data available regarding pre- and post- $\mathrm{PCl}$ serum creatinine. Data were collected regarding demographics, clinical characteristics, co-morbidities, laboratory data and $\mathrm{PCl}$ data. The MR score was calculated for each patient who were then stratified into four groups: MR score 0-5 (group 1), 6-10 (group 2), 11-15 (group 3), and $\geq 16$ (group 4). Patients could have slightly different MR score based on whether the serum creatinine or estimated glomerular filtration rate was used.

\section{Clinical definitions and follow-up}

CIAKI was defined as an increase in serum creatinine of $\geq 25 \%$ or $\geq 0.5 \mathrm{mg} / \mathrm{dl}$ over baseline at 48 hours post$\mathrm{PCl}$. Chronic kidney disease was defined as baseline serum creatinine $>1.5 \mathrm{mg} / \mathrm{dl}$ or an estimated glomerular filtration rate $<60 \mathrm{ml} / \mathrm{min} / 1.73 \mathrm{~m}^{2}$ (Levey modified 'Modification of Diet in Renal Disease formula). Anemia
Table 2: Baseline demographics, comorbidities, and procedural characteristics.

\begin{tabular}{|c|c|}
\hline Variable & $\begin{array}{l}\text { Value or Frequency } \\
(n=931)\end{array}$ \\
\hline Age (yrs) (median, IQR) & $65(56-75)$ \\
\hline Age $>75$ & $23.6 \%$ \\
\hline Caucasian & $92.5 \%$ \\
\hline Male & $68.1 \%$ \\
\hline Diabetes mellitus & $37.9 \%$ \\
\hline Hypertension & $83.7 \%$ \\
\hline Hyperlipidemia & $77.7 \%$ \\
\hline Smoking history & $52.2 \%$ \\
\hline Congestive heart failure & $11.1 \%$ \\
\hline Hypotension & $1.3 \%$ \\
\hline Previous myocardial infarction & $30.8 \%$ \\
\hline Previous CABG & $24.4 \%$ \\
\hline Peripheral vascular disease & $13.1 \%$ \\
\hline Previous angioplasty & $37.6 \%$ \\
\hline Hematocrit (\%) (median, IQR) & $40(37-43)$ \\
\hline Anemia & $26.9 \%$ \\
\hline $\begin{array}{l}\text { Baseline serum } \mathrm{Cr}(\mathrm{mg} / \mathrm{dl}) \text { (median, } \\
\text { IQR) } \\
\quad<1.5 \\
1.5-2.0 \\
>2.0\end{array}$ & $\begin{array}{l}1.0(0.9-1.2) \\
85.5 \% \\
11.7 \% \\
2.8 \%\end{array}$ \\
\hline $\begin{array}{l}\text { Baseline eGFR }\left(\mathrm{ml} / \mathrm{min} 1.73 \mathrm{~m}^{2}\right) \\
\text { (median, IQR) }\end{array}$ & $73(57-89)$ \\
\hline$>60$ & $69.8 \%$ \\
\hline $40-60$ & $20.8 \%$ \\
\hline $20-40$ & $9.1 \%$ \\
\hline$<20$ & $0.2 \%$ \\
\hline Multivessel coronary artery disease & $60.6 \%$ \\
\hline Multivessel PCl & $23.7 \%$ \\
\hline Treated saphenous vein graft & $7.0 \%$ \\
\hline Intra-aortic balloon pump & $1.3 \%$ \\
\hline Contrast volume (ml) (median, IQR) & $193(135-258)$ \\
\hline Contrast volume $>150 \mathrm{ml}$ & $67.1 \%$ \\
\hline $\begin{array}{l}\text { Pre-treated with intravenous sodium } \\
\text { bicarbonate }\end{array}$ & $3.4 \%$ \\
\hline Pre-treated with $\mathrm{N}$-acetycysteine & $15.2 \%$ \\
\hline
\end{tabular}

was defined using World Health Organization criteria: baseline hematocrit value $<39 \%$ for men and $<36 \%$ for women. Hypotension was defined as systolic blood pressure $<80 \mathrm{mmHg}$ for at least one hour requiring inotropic support with medications or intra-aortic balloon pump within 24 hours following PCI. Renal replacement therapy (RRT) initiation was assessed within one month after $\mathrm{PCl}$. All-cause mortality was assessed within one year after $\mathrm{PCl}$ and was ascertained using hospital medical records or the Social Security Death Index at http://ssdi.rootsweb.ancestry.com 


\section{Statistical analysis}

Summary statistics for the demographic, clinical, laboratory, and procedural characteristics of the population were computed. The proportions of patients who had each of the outcomes (CIAKI, renal replacement therapy and death) were calculated for the entire population and for each MR score subgroup. Continuous variables were compared between patients with and without outcomes using ANOVA or Kruskal-Wallis as appropriate. Discrete variables were compared using Chi Square and Fisher's Exact as appropriate. In order to assess MR score discrimination, likelihood ratios (LR) were calculated for each MR score group within our dataset and also for the dataset reported in Mehran, et al. [10]. A Chi Square for trend was used to compare the occurrence of CIAKI, RRT and death by MR score group. All calculations were performed using Stata, Release 11, College Station, TX.

\section{Results}

After applying inclusion and exclusion criteria a total of 931 consecutive patients from the three different hospitals were included in the study. Table 2 details baseline demographics, clinical characteristics, laboratory and $\mathrm{PCl}$ data. Overall, the mean age was 65 (mean \pm SD) years and the population was $68.1 \%$ male and $92.5 \%$ Caucasian. Compared to Mehran, et al. our population had a higher rate of most co-morbidities including hypertension ( $83.7 \%$ vs. $62.1 \%)$, diabetes mellitus (37.9\% vs. $30.7 \%)$, and congestive heart failure (11.1\% vs. 6.0\%). However, our population had lower rates of prior myocardial infarction ( $30.8 \%$ vs. $53.4 \%)$, previous revascularization with angioplasty $(37.6 \%$ vs. $49.4 \%)$ or coronary artery bypass graft $(24.4 \%$ vs. $39.9 \%)$, hypotension ( $1.3 \%$ vs. $8.3 \%)$, and use of intraaortic balloon pump (1.3\% vs. $7.1 \%)$. Our population had a higher rate of multi-vessel coronary artery disease (60.6\% vs. $26.9 \%)$. Our population had lower amounts of intravenous contrast used (median $193 \pm 123 \mathrm{ml}$ vs. $261 \pm 122 \mathrm{ml}$ ). The baseline serum creatinine of our cohort was similar to that of Mehran, et al. $1.0 \pm 0.3 \mathrm{mg} /$ $\mathrm{dl}$, eGFR $73 \pm 16$ and $30.2 \%$ had CKD vs. eGFR was $72.7 \pm$ 21.1 and $26.4 \%$ had CKD, respectively.

The overall incidence of CIAKI was $12.2 \%$, similar to the $13.1 \%$ event rate in the development dataset of
Mehran, et al. The overall rate of RRT within one month of $\mathrm{PCl}$ was $0.4 \%$ in our population compared to $0.6 \%$ in Mehran, et al. report. The overall rate of death at one year was $9.0 \%$ compared to $6.0 \%$ in Mehran, et al. report.

As detailed previously, patients were stratified into a low-risk group (Group 1, MR score 0-5, $n=508,54.6 \%$ ), moderate risk group (Group 2, MR score 6-10, $n=283$, $30.4 \%$ ), high risk group (Group 3, MR score 11-15, n $=114,12.2 \%$ ), and very high-risk group (Group 4, MR score $\geq 16, n=26,2.8 \%$ ). Overall, our population skewed towards a higher MR score than the original derivation dataset from Mehran, et al. (59.2\% in MR score group 1, $31.7 \%$ in MR score group 2, 7.9\% in MR score group 3, and $1.1 \%$ in MR score group 4).

Table 3 details the event rates and respective likelihood ratios stratified by MR score group. A higher MR score was strongly associated with development of CIAKI and mortality $(p<0.01)$. The LR ratios for MR score group 4 were most predictive of CIAKI, RRT, and one- year all-cause mortality, with LR of 7.2, 19.3, and 6.3 , respectively. Conversely, the LR for MR score group 1 were moderately predictive against those outcomes, with $L R$ of $0.4,0$, and 0.3 , respectively. The $L R$ for $M R$ score group 2 were not predictive of CIAKI, RRT, and one-year all-cause mortality, with LR between 0.8 and 1.1. The $L R$ for $M R$ score group 3 were mildly predictive of the outcomes, with LR between 2.1 and 4.3.

Table 3 also compares our results with those previously reported by Mehran, et al. There was no difference in the rate of CIAKI overall or in each MR score group when the two populations were compared. However, the risk of death was higher in our population (RR 1.58, 95\% Cl: 1.37 to $1.89, p<0.001$ ). The likelihood ratios between the two populations were quite similar overall.

\section{Discussion}

Angiography remains the gold standard for the diagnosis and management of CAD. Chronic kidney disease is prevalent in patients with CAD. The coexistence of these conditions puts patents at higher risk for CIAKI related to nephrotoxic contrast exposure [1]. The MR score was proposed to help identify patients undergoing non-emergent $\mathrm{PCl}$ at highest risk for CIAKI and death related to contrast exposure.

Table 3: Outcomes and likelihood ratios.

\begin{tabular}{|l|l|l|l|l|l|l|l|l|}
\hline & \multicolumn{3}{l}{} & \multicolumn{2}{l}{ MRS Group 1 } & \multicolumn{2}{l|}{ MRS Group 2 } & \multicolumn{2}{l|}{ MRS Group 3 } \\
\cline { 2 - 9 } & Reuter & Mehran & Reuter & Mehran & Reuter & Mehran & Reuter & Mehran \\
\hline Patients (n) & 508 & 2486 & 283 & 1633 & 114 & 599 & 26 & 154 \\
\hline CIN (\%) & 5.7 & 7.5 & 13.1 & 14 & 30.7 & 26.1 & 50 & 57.3 \\
\hline HD (\%) & 0 & 0.04 & 0.4 & 0.12 & 0.9 & 1.09 & 7.7 & 12.6 \\
\hline LR CIN & 0.4 & 0.5 & 1.1 & 1.0 & 3.2 & 2.3 & 7.2 & 8.5 \\
\hline LR HD & 0 & 0.07 & 0.8 & 0.2 & 2.1 & 2.0 & 19.3 & 23.5 \\
\hline LR Mortality & 0.3 & 0.3 & 0.9 & 1.0 & 4.3 & 3.0 & 6.3 & 7.5 \\
\hline
\end{tabular}


Our study, designed to externally validate MR score, was conducted at 3 centers $(2$ university teaching hospitals in geographically different areas of the country and 1 community-based teaching hospital). When compared to the patient characteristics in Mehran study, our cohort had a higher prevalence of several co-morbidities. Despite these differences, MR score accurately predicted the risk of CIAKI and one-year allcause mortality. Patients with high MR score (group 4) were found to have $>10$-fold increase incidence of $\mathrm{CIAKI}$ and one-year all-cause mortality when compared to those with low MR score (group 1). Although fewer of our patients required RRT than in the Mehran study, we did find that there was a greater likelihood of RRT in patients with higher MR scores. The lower need for RRT in our cohort might be explained by different thresholds for initiation of renal replacement therapy at different institutions. The higher mortality rates in our cohort compared to the Mehran, may reflect the higher prevalence of co-morbidities such as diabetes mellitus and congestive heart failure in our cohort. Moreover, because our study was retrospective, serum creatinine values 48-72 hours post $\mathrm{PCl}$ were only available in hospitalized patients who tend to have more risk factors than patients undergoing elective $\mathrm{PCl}$. Prospective collection of data in Mehran's study may have captured more post- $\mathrm{PCl}$ serum creatinine values in patients undergoing elective outpatient procedures.

We found that MR score is a valid tool that can be used by clinicians to identify patient populations that are at risk for $\mathrm{CIAKI}$ following non-emergent $\mathrm{PCI}$. There have been other validation studies published previously. Sgura and coworkers applied the MR score to predict the risk of $\mathrm{CIAKI}$ in patients undergoing $\mathrm{PCI}$ for acute ST elevation myocardial infarction (STEMI) $[12,13]$. Patients with a low-risk score had a slightly higher incidence of CIAKI (14.4\%) compared to those with a high-risk score (14.2\%), and a there was a 10 -fold increase in one-year all cause mortality in the very highrisk patients as compared to a 6 -fold increase in patients with a low-risk score. This study only included patients with STEMI, which may explain the higher morbidity and mortality rates that were reported. Recently R.A. Abella's-Sequeiros, et al. reported a validation study [14] that showed an overall incidence of CIAKI of $7.8 \%$ which was significantly lower than our study. The rate of CIAKI increased with increased risk category $2.4 \%, 7.2 \%$, $18.6 \%, 40 \%$ for low, moderate, high and very high-risk groups. Compared to our cohort, their population was older but had lower prevalence of diabetes mellitus, peripheral vascular disease and prior $\mathrm{MI}$, which could explain the lower incidence of CIAKI. Moreover, their study included patients from one center in Europe whereas our study included patients from three different centers. Our study is the first multicenter validation of the MR score in heterogeneous population undergoing non-emergent $\mathrm{PCl}$.
CIAKI is an important complication of coronary angiography and $\mathrm{PCI}$. In order to give informed consent for the procedure, patients and families need to understand its risks. Merely mentioning the development of CIAKI, along with the impact of hemodialysis on the quality of life, might make patients reluctant to undergo a potentially lifesaving procedure. Accordingly, accurate risk stratification with hard end points provides patients with a valid and accurate means for making decisions. Hopefully, we can reduce the risk of CIAKI by identifying the patients at high risk and implementing preventive measures. $[7,15,16]$. As such, predictive scores such as MR score can be of substantial benefit for both clinical decision-making and research.

Our study has several limitations. Variables which may impact CIAKI such as peri-procedural hydration volume, proteinuria, and nephrotoxic medications $[17,18]$ were not included in our analysis since our goal was to validate MR score and these variables were not considered in the Mehran study. Similarly, although we collected information regarding $\mathrm{N}$-acetyl cysteine and sodium bicarbonate administration, we did not include them in risk prediction since they were not considered in Mehran's study and the role of these interventions in preventing CIAKI has not been clearly defined $[19,20]$. Approximately $60 \%$ of patients in our cohort had multivessel coronary disease, however less than $25 \%$ of cohort patients underwent multivessel $\mathrm{PCl}$, which suggests that coronary artery bypass rates may be higher in our population than in Mehran's cohort. Our study, like other studies evaluating prognostic impact of CIAKI, fails to confirm whether CIAKI is a causative factor in post $\mathrm{PCl}$ mortality or whether it is simply a marker of patients with multiple co-morbidities [21]. A prospective randomized trial would conclusively answer that question. Although ours was a retrospective study which introduced variability in the timing of post- $\mathrm{PCl}$ follow up serum creatinine measurements, our results were comparable to those of Mehran, et al.

In conclusion, we were able to externally validate MR score as a useful tool in predicting the risk of CIAKI and death following non-emergent $\mathrm{PCl}$ in a heterogeneous population at three different hospitals. The MR score can help clinicians identify and stratify patients according to risk for CIAKI. Because interventions may be variably effective in different risk groups, the MR score may also aid researchers stratify patients into different risk groups for future CIAKI prevention studies.

\section{Funding}

None.

\section{References}

1. McCullough PA (2008) Contrast-induced acute kidney injury. J Am Coll Cardiol 51: 1419-1428.

2. Dangas G, lakovou I, Nikolsky E, Aymong ED, Mintz GS, et al. (2005) Contrast-induced nephropathy after 
percutaneous coronary interventions in relation to chronic kidney disease and hemodynamic variables. Am J Cardiol 95: 13-19.

3. Marenzi G, Lauri G, Assanelli E, Campodonico J, Metrio MD, et al. (2004) Contrast-induced nephropathy in patients undergoing primary angioplasty for acute myocardial infarction. J Am Coll Cardiol 44: 1780-1785.

4. Subramanian S, Tumlin J, Bapat B, Zyczynski T (2007) Economic burden of contrast-induced nephropathy: Implications for prevention strategies. J Med Econ 10: 119134.

5. Rihal CS, Textor SC, Grill DE, Berger PB, Ting $\mathrm{HH}$, et al. (2002) Incidence and prognostic importance of acute renal failure after percutaneous coronary intervention. Circulation 105: 2259-2264.

6. Levy EM, Viscoli CM, Horwitz RI (1996) The effect of acute renal failure on mortality. A cohort analysis. JAMA 275: 1489-1494.

7. Nash K, Hafeez A, Hou S (2002) Hospital-acquired renal insufficiency. Am J Kidney Dis 39: 930-936.

8. Kelly AM, Dwamena B, Cronin P, Bernstein SJ, Carlos RC (2008) Meta-analysis: Effectiveness of drugs for preventing contrast-induced nephropathy. Ann Intern Med 148: 284294.

9. Kshirsagar AV, Poole C, Mottl A, Shoham D, Franceschini $\mathrm{N}$, et al. (2004) $\mathrm{N}$-acetylcysteine for the prevention of radiocontrast induced nephropathy: $A$ meta-analysis of prospective controlled trials. J Am Soc Nephrol 15: 761769.

10. Mehran R, Aymong ED, Nikolsky E, Lasic Z, lakovou I, et al. (2004) A simple risk score for prediction of contrast-induced nephropathy after percutaneous coronary intervention: Development and initial validation. $\mathrm{J}$ Am Coll Cardiol 44: 1393-1399.

11. Candela-Toha A, Elias-Martin E, Abraira V, Tenorio MT, Parise D, et al. (2008) Predicting acute renal failure after cardiac surgery: External validation of two new clinical scores. Clin J Am Soc Nephrol 3: 1260-1265.
12. Sato A, Hoshi T, Kakefuda $Y$, Harunari $T$, Watabe $H$, et al. (2015) Effect of the mehran risk score for the prediction of clinical outcomes after percutaneous coronary intervention. J Cardiol 66: 417-422.

13. Sgura FA, Bertelli L, Monopoli D, Leuzzi C, Guerri E, et al. (2010) Mehran contrast-induced nephropathy risk score predicts short- and long-term clinical outcomes in patients with ST-elevation-myocardial infarction. Circ Cardiovasc Interv 3: 491-498.

14. Abellás-Sequeiros RA, Raposeiras-Roubín S, Abu-Assi E, González-Salvado V, Iglesias-Álvarez D, et al. (2016) Mehran contrast nephropathy risk score: Is it still useful 10 years later? J Cardiol 67: 262-267.

15. Nikolsky E, Mehran R, Turcot D, Aymong ED, Mintz GS, et al. (2004) Impact of chronic kidney disease on prognosis of patients with diabetes mellitus treated with percutaneous coronary intervention. Am J Cardiol 94: 300-305.

16. McCullough PA, Adam A, Becker CR, Davidson C, Lameire $\mathrm{N}$, et al. (2006) Epidemiology and prognostic implications of contrast-induced nephropathy. Am J Cardiol 98: 5K-13K.

17. Perazella MA, Reilly RF (2011) Imaging patients with kidney disease: How do we approach contrast related toxicity? Am J Med Sci 341: 215-221.

18. McCullough PA, Wolyn R, Rocher LL, Levin RN, O'Neill WW (1997) Acute renal failure after coronary intervention: Incidence, risk factors, and relationship to mortality. Am J Med 103: 368-375.

19. Nikolsky E, Mehran R, Lasic Z, Mintz GS, Lansky AJ, et al. (2005) Low hematocrit predicts contrast-induced nephropathy after percutaneous coronary interventions. Kidney Int 67: 706-713.

20. Marenzi G, Assanelli E, Campodonico J, Lauri G, Marana I, et al. (2009) Contrast volume during primary percutaneous coronary intervention and subsequent contrast-induced nephropathy and mortality. Ann Intern Med 150: 170-177.

21. Bartorelli AL, Marenzi G (2008) Contrast-induced nephropathy. J Interv Cardiol 21: 74-85. 